\title{
Sodium-azide-induced learning deficits in rats: Time course and corresponding pathology
}

\author{
SUSAN D. CROLL, NEFERTITI A. GREENE, RONALD M. LINDSAY, and STANLEY J. WIEGAND \\ Regeneron Pharmaceuticals Inc., Tarrytown, New York
}

\begin{abstract}
Systemic administration of sodium azide, a selective inhibitor of the mitochondrial enzyme cytochrome oxidase, has been reported to produce deficits in memory and long-term potentiation (Bennett, Diamond, Stryker, Parks, \& Parker, 1992; Bennett \& Rose, 1992). We have further characterized the memory deficits observed during the period of infusion of this toxin and have evaluated learning and memory following the termination of infusions to assess the permanence of deficits. In addition, the brains of sodium-azide-infused rats were examined histologically to evaluate neuropathological changes that might accompany the learning deficits. We confirmed the deficit in water-maze acquisition observed previously during sodium-azide infusion (Bennett \& Rose, 1992) but found that azideinfused animals were as capable as vehicle-infused animals of remembering the platform location once they had learned it. In addition, during sodium-azide infusion, we found a short-term passive-avoidance retention deficit that slowly improved with longer delays. Sodium-azide-infused animals showed deficits in water-maze reversal retention shortly after the infusion was terminated, but they recovered by 6 months postinfusion. No significant locomotor differences were detected between azide- and vehicleinfused animals, but a significant fine sensorimotor deficit was detected during azide infusion. Histological analyses revealed no obvious neuronal cell loss after sodium-azide infusion, but rather a loss of myelinated fiber staining in the retrosplenial (posterior cingulate) cortex. The sodium-azide-infused rat may thus serve as an animal model of learning deficits resulting from metabolic and structural alterations in the limbic cortex.
\end{abstract}

Metabolic stress has been suggested as an etiological factor for many neurological disorders, especially those that have age as a risk factor (Beal, Hyman, \& Koroshetz, 1993). This idea proposes that prolonged exposure to metabolic stress throughout life results in mitochondrial dysfunction leading to neurodegenerative changes. Among these disorders is Alzheimer's disease (AD), for which age is a primary risk factor (for review, see Jorm, Korten, \& Henderson, 1987).

Sodium azide infused subcutaneously in rats for 28 days induces metabolic stress characterized by a selective depletion of cytochrome oxidase (Complex IV) (Bennett, Diamond, Stryker, Parks, \& Parker, 1992). Accompanying this reduction in cytochrome oxidase are impairments in radial arm maze and two-way active-avoidance acquisition (Bennett et al., 1992). Because AD is characterized by both memory impairments (for review, see Carlesimo \& Oscar-Berman, 1992) and reduced or altered levels of cytochrome oxidase (Kish et al., 1992; Parker, Filley, \& Parks, 1990; Simonian \& Hyman, 1993), Bennett et al. (1992) have proposed that the sodium-azide-infused rat may serve as an animal model of certain aspects of $\mathrm{AD}$.

\footnotetext{
The authors wish to thank Catherine R. Chesnutt for technical assistance and Nancy Stambler for statistical advice. Correspondence should be addressed to S. D. Croll, Regeneron Pharmaceuticals Inc., 777 Old Saw Mill River Rd., Tarrytown, NY 10591 (e-mail: susan_ croll@regpha.com).
}

-Accepted by previous editor, Paul E. Gold
To explore this possibility, we infused rats with sodium azide to further characterize the extent and duration of memory impairments and to examine the hippocampus and cortex for evidence of histopathological changes. Bennett and Rose (1992) reported deficits in both watermaze acquisition and water-maze retention measured by a spatial probe trial. However, the azide-infused animals had never fully acquired the task, as measured by latency to escape, before being tested for retention of the escape platform location in the spatial probe trial. In the present study, we used a water-maze acquisition protocol that allowed for more rapid acquisition of the escape platform location, so that we could test retention after the task had been fully acquired by both vehicle- and azide-infused animals. In addition, we tested retention deficits at different delays after one-trial acquisition of a passive-avoidance task. To assess the permanence of azide-induced learning and memory deficits, we also examined learning and memory after termination of the azide infusions.

Our results confirm the previous finding of learning impairments during sodium-azide infusion (Bennett et al., 1992; Bennett \& Rose, 1992), but we find that once a task has been acquired, azide-infused animals are able to demonstrate retention of what they learned. We also find that animals continue to show some degree of transient memory impairment after the azide infusion has ended. Finally, we observe that these impairments are accompanied by a reduction of myelinated fiber staining in the retrosplenial (posterior cingulate) limbic cortex. The retrosplenial cortex has previously been shown to be in- 
volved in learning and memory in both laboratory animals and humans (Berger, Weikart, Bassett, \& Orr, 1986; Gabriel, 1990; Molchan, Sunderland, McIntosh, Herscovitch, \& Schreurs, 1994; Sutherland \& Hoesing, 1993; Swartz, Halgren, Fuster, \& Mandelkern, 1994; Tulving, Markowitsch, Kapur, Habib, \& Houle, 1994).

\section{EXPERIMENT 1}

To further describe and characterize learning and memory deficits that occur during sodium-azide administration, animals were behaviorally evaluated in the water maze and on a passive-avoidance task. Previous work (Bennett \& Rose, 1992) showed water-maze acquisition and retention deficits, but retention was tested before animals had fully acquired the task as measured by latency to escape. In Experiment 1, animals were tested for a retention deficit once they had demonstrated good performance of the water-maze task. In addition, we used a one-trial passive-avoidance task to expand the observation of active-avoidance deficits (Bennett et al., 1992) to passive-avoidance protocols.

\section{Method}

\section{Subjects}

Adult male Sprague-Dawley rats $(350-500 \mathrm{~g}$ at the start of the experiment) were used as subjects for these experiments. The rats were maintained in an environmentally controlled animal colony with a 12:12-h light:dark cycle (lights on $0600 \mathrm{~h}$ ). Food and water were available ad lib. All animal experiments were conducted under a protocol reviewed and approved by an Institutional Animal Care and Use Committee following NIH guidelines.

\section{Surgeries}

The animals were anesthetised with ketamine/xylazine anesthesia $(0.5 \mathrm{ml} / \mathrm{kg} 25 \%$ ketamine and $0.1 \mathrm{ml} / \mathrm{kg} 5 \%$ xylazine i.p.) prior to implantation or removal of Alzet 28-day osmotic minipumps (Model 2ML4, Alza Corp., Palo Alto, CA). Pumps were implanted subcutaneously along each animal's right flank and contained either sodium azide ( $400 \mu \mathrm{g}$ per $2.5 \mu \mathrm{l}$ per hour) dissolved in $0.9 \%$ sterile saline or $0.9 \%$ sterile saline vehicle $(2.5 \mu 1$ per hour). Relative to vehicle-infused rats, sodium-azide-infused rats remained in good general health throughout the study. All rats gained weight, and, at the end of infusions, the average weight of azide-infused rats was only $3 \%$ less than that of vehicle-infused rats (a nonsignificant difference).

\section{Behavioral Testing}

Behavioral data were collected during the 3 rd and 4 th weeks of infusion. All behavioral testing was performed between 1000 and $1600 \mathrm{~h}$ in behavioral testing rooms located within the animal facility. Whenever a behavioral task ran across multiple days, the animals were tested within $1 \mathrm{~h}$ of the same time each day. The animals were placed either in the testing room or in the room's holding area for approximately $1 \mathrm{~h}$ before testing, in order to allow acclimation to the testing environment before evaluation. Figure 1 shows the behavioral testing protocols for Experiment 1.

Passive avoidance. Behaviorally naive animals were exposed to a one-trial passive-avoidance learning paradigm using an automated two-chamber shuttlebox system (Coulbourne Instruments, Allentown, PA), which delivered a $1-\mathrm{mA}, 5-\mathrm{sec}$ footshock to the animals after they crossed from the undesirable brightly lit chamber into the more desirable dark chamber. In order to assess retention of the footshock at $30 \mathrm{~min}, 24 \mathrm{~h}, 48 \mathrm{~h}, 72 \mathrm{~h}$, and 1 week following the ini- tial acquisition, the animals were placed into the light chamber, and the latency for the animals to cross into the dark chamber was measured. During all trials, a guillotine door closed after the animal crossed into the dark chamber, forcing the animal to remain on the dark side until removed from the apparatus. Any animal that had not crossed into the dark chamber by $5 \mathrm{~min}$ after initiation of the trial was assigned a maximum trial latency of $300 \mathrm{sec}$ and was removed from the shuttlebox. No shocks were delivered to the animals upon crossing into the dark chamber during the retention trials. Because many animals achieved the maximum score of $300 \mathrm{sec}$, passive-avoidance retention was analyzed at each delay with a nonparametric Mann-Whitney $U$ test to determine whether the azideinfused animals and the vehicle-infused animals performed differently. Passive-avoidance testing was performed during the $3 \mathrm{rd}$ week of azide infusions.

The latency to the first crossover (during the acquisition trial) was used as a control for spontaneous tendency to cross over. A Student's independent-groups $t$ test was used to examine the difference in initial crossover latency between vehicle- and azide-infused animals.

A hot-plate test was used to make certain that experimental animals were not analgesic relative to controls. If experimental animals were found to be analgesic, any decrease in their latency to cross over might be attributable to a decreased sensitivity to the pain associated with footshock.

Hot-plate test. The animals received one trial on the hot-plate test on Day 25 or 26 of infusion, to assess their pain threshold. The animals were placed on a $50^{\circ}-52^{\circ} \mathrm{C}$ hot plate enclosed in a clear Plexiglas cylinder. Timing was started the instant the animal's paws touched the hot plate and was terminated when the animal either made an escape attempt or licked its hindpaws. A Student's independentgroups $t$ test was used to compare the hot-plate latencies of vehicleand azide-infused rats.

Water-maze acquisition. Water-maze acquisition was tested using a modification of Morris's water-maze task (Morris, 1981) which allowed the animals to learn the task in fewer trials. The animals received one trial per day for 6 days, with each trial starting from a pseudorandomly selected starting point along the perimeter of the water maze (the starting point changed each day). The animals that did not find the hidden escape platform within 5 min were guided to the platform by hand and were assigned an escape latency of $300 \mathrm{sec}$. The prolonged trial time used in this protocol increased exploration time and motivation to find the platform, and the 24-h intertrial interval allowed for sensitive detection of acquisition deficits. The animals were tested for water-maze acquisition ability beginning the 3 rd week of infusions. All animals were behaviorally naive when tested. A 6 (trial) $\times 2$ (infusion) mixed factorial analysis of variance (ANOVA) was performed to compare learning in the azide-infused groups and that in the vehicle-infused controls, and a Tukey HSD post hoc test was used to further evaluate differences between groups.

Water-maze retention. Water-maze retention was tested with a spatial probe trial in which the animal was placed in the water maze with the escape platform removed. Each animal was started in the center of the water maze, and the time it spent in each quadrant was measured during a $30-\mathrm{sec}$ trial. Retention of the spatial location of the escape platform was represented by the proportion of time spent in the quadrant that had previously contained the escape platform. Student's $t$ test for independent groups was used to compare retention scores for vehicle- and azide-infused animals.

Water-maze controls. Two different cue-learning tasks were used to evaluate whether any acquisition deficits were specific to spatial learning or whether they were due to sensory nonspatial learning or motivational factors (Morris, 1981). The naive animals were trained to locate a platform signaled by a cue in the water maze. A vertically striped cue card was placed on the wall of the maze adjacent to the hidden platform. The animals received six daily acquisition trials, similar to those described for the spatial condition. The animals also received a retention trial in which the 
A

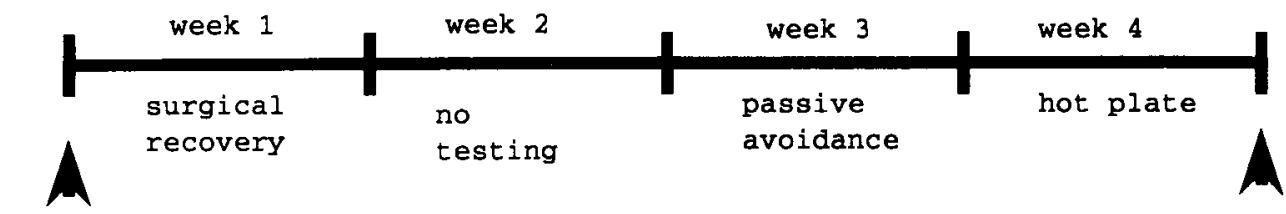

start of

sacrifice

infusions

B

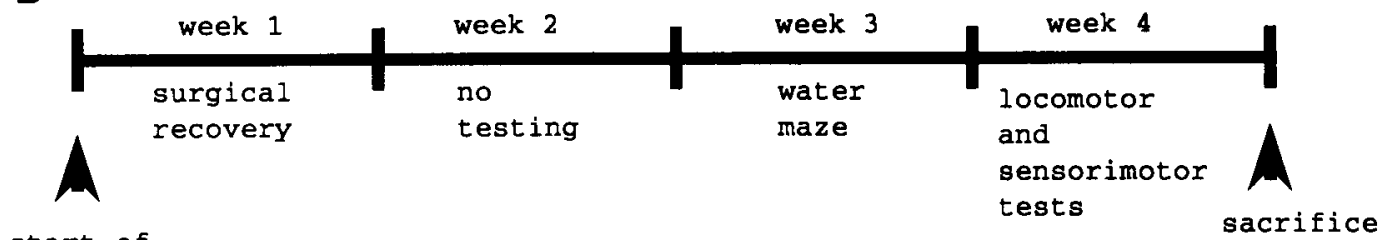

start of

infusions

C

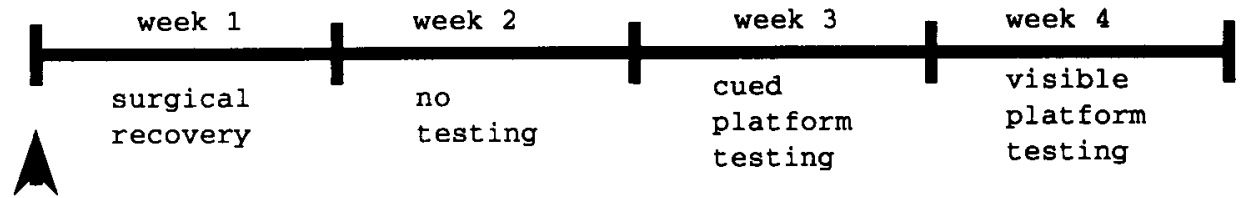

start of

infusions

Figure 1. Time lines for behavioral evaluation of animals during azide infusions. (A) Passive-avoidance animals. (B) Water-maze animals. (C) Water-maze controls.

platform was removed, but the cue card remained. This retention trial was conducted as described for the spatial probe trial. In addition, the animals received one trial in which they were placed into the water maze cued by a visible escape platform. Latency to escape to the visible platform was measured. Cued-platform acquisition and retention were analyzed as described for the spatial acquisition and retention tasks. Latency to escape to a visible platform was analyzed using a Student's independent-groups $t$ test.

Locomotion. The animals were placed on a $3 \times 3 \mathrm{ft}$ square table divided into nine 1 -ft square grids. The number of grids crossed by each animal was measured during a 4-min trial on Day 26 or 27 of the infusion. A Student's independent-groups $t$ test was used to compare the number of grids crossed by vehicle- and azideinfused rats.

Sensorimotor integration tests. The animals were evaluated for fine sensorimotor integration and motor coordination by determining whether or not they were able to grasp a Plexiglas rod ( $0.25 \mathrm{in}$. in diameter) in their forepaws. Gross sensorimotor integration was tested by determining whether or not the animals were able to reach out for an approaching flat-surfaced edge with their forepaws and hindpaws. Because testing these skills only provided a means of detecting severe deficits that might interfere with other behavioral tasks, the animals were scored either "yes" or "no" on each task, simply indicating whether or not they were able to perform the skill. Fisher's exact test for contingency tables was conducted for each of these measures to compare the sensorimotor performance of vehicle- and azide-infused rats. The animals were tested for sensorimotor ability on Day 26 or 27 of infusions.
Histology. Brain tissue was collected for histological processing on Day 27 or 28 of infusions. The animals were deeply anesthetised with an i.p. injection of ketamine/xylazine, exsanguinated, and perfused transcardially with heparinized saline followed by $4 \%$ paraformaldehyde in acetate buffer ( $\mathrm{pH} 6.5$ ) and then $4 \%$ paraformaldehyde in borate buffer ( $\mathrm{pH} 9.5$ ). After fixation by perfusion, brains were removed and postfixed overnight with $4 \%$ paraformaldehyde in borate buffer. Brains were then transferred into a solution of $30 \%$ sucrose in borate buffer, where they remained for 3-7 days until sectioned. Brains were frozen and sectioned coronally at $30 \mathrm{mi}-$ crons on a sliding microtome. Half of the sections from each brain were stored at $4^{\circ} \mathrm{C}$ in $10 \%$ formalin, and the other half were stored at $-20^{\circ} \mathrm{C}$ in cryoprotectant (Watson, Wiegand, Clogh, \& Hoffman, 1986) until stained.

Sections stained for Nissl substance were taken from those stored at $-20^{\circ} \mathrm{C}$ in cryoprotectant. They were first washed in PBS and mounted onto slides coated with $4 \%$ gelatin. Sections were then defatted for $90 \mathrm{~min}$ in a 1:1 chloroform:ethanol solution. After hydration through graded ethanols, sections were stained with thionin, rinsed in double-distilled water, dehydrated through graded ethanols, and cleared in xylenes. Visual examination of Nissl-stained sections by a blind evaluator was used to screen the entire brain for any obvious areas of cell loss.

Sections were stained with a silver stain used to visualize normal myelinated fibers (Blackstad, Heimer, \& Mugnaini, 1981). To ensure adequate fixation, all sections used for the silver stain were taken from those stored in formalin. Free-floating sections were incubated for $5 \mathrm{~min}$ in a $10 \%$ silver nitrate solution and then rinsed in 
absolute ethanol. Sections went through two series of incubation in an ammoniacal silver nitrate solution, reduction in Nauta-Gygax reducer, and differentiation with potassium ferricyanide. After fixation with $1 \%$ sodium thiosulfate, sections were transferred to phosphate-buffered saline overnight and mounted the next day. All silver-stain runs contained both vehicle- and azide-infused brains to ensure that variability in silver impregnation would be represented across the experimental conditions.

Visual inspection of silver-stained sections indicated that myelinated fiber density in the retrosplenial cortex was specifically affected by azide treatment. To confirm this observation, fiber density in the retrosplenial cortex was quantified using the Loats Ras-Cal-Plus Image Analysis System (Westminster, MD). To control for variability between brains and between different runs of silver stains, fiber density was also quantified in an unaffected area of cortex in the same section. Specifically, the optical density of an approximately $0.5-\mathrm{mm}$ strip of the entire depth of granular retrosplenial cortex was measured for myelinated fiber density bilaterally. In addition, an approximately $0.5-\mathrm{mm}$ strip of the entire depth of cortex sampled from Area 2 of the occipital cortex was measured in the same section bilaterally. The mean density of retrosplenial cortex from each animal's samples was then normalized to the mean density of the occipital cortex samples to arrive at a normalized density for each animal. Normalized densities from vehicle- and azideinfused animals were compared using a Student's independentgroups $t$ test.

\section{Results}

\section{Behavior}

Passive avoidance. Azide-infused animals showed a significant passive-avoidance retention deficit, but, as the delay between acquisition and retention increased, the azide-infused animals' performance improved to the level of vehicle-infused animals. Specifically, the retention deficit was statistically significant at $30 \mathrm{~min}$ postacquisition $[U(11,12)=90, p<.05$, one-tailed] but was no longer significant by $24 \mathrm{~h}, 48 \mathrm{~h}$, and 1 week postacquisition $[U(11,12)=68,66$, and 62.5 , n.s.; Figure $2 \mathrm{~A}]$. It was unlikely that performance factors alone caused the temporary deficit, because azide-infused animals spontaneously crossed over to the dark chamber only slightly faster than did vehicle-infused animals $[t(21)=1.905$, $p>.07$; Figure 2B]. In addition, the pain threshold of azide-infused animals was no different from that of vehicle-infused animals as measured by the hot-plate test $[t(21)=1.057, p>.30$; Figure $2 \mathrm{C}]$.

Water maze. During sodium-azide infusion, there was a significant water-maze acquisition deficit in sodiumazide-infused rats relative to vehicle-infused rats $[F(1,13)$ $=6.012, p<.03$; Figure 3A]. However, after learning the platform location, the animals demonstrated no deficit in platform-location retention, as determined by the spatial probe trial $[t(13)=0.909, p>.90$; Figure $3 \mathrm{~B}]$. The water-maze acquisition deficit was not likely to be caused by sensory, motivational, or performance factors, because azide-infused animals learned to swim to a hidden platform located adjacent to a cue card at least as quickly as vehicle-infused animals $[F(1,10)=2.193, p>$ .17 ; Figure $3 \mathrm{C}]$. In addition, they were not significantly different from vehicle-infused animals at searching near the cue card when the platform had been removed $[t(10)=$ $1.462, p>.17$; data not shown]. Finally, azide-infused rats escaped onto a visible platform as quickly as vehicleinfused rats $[t(10)=0.654, p>.52$; Figure 3D].

Locomotion and sensorimotor integration. Gridcrossing measures revealed no significant difference in locomotor activity between vehicle- and azide-infused rats during infusion $[t(13)=1.43, p>.17$; Figure 4]. In addition, rats from both groups were able to demonstrate normal gross sensorimotor coordination by reaching for an edge with their paws. In contrast, we observed a significant deficit in fine sensorimotor coordination during azide infusion. Significantly fewer animals infused with
A.

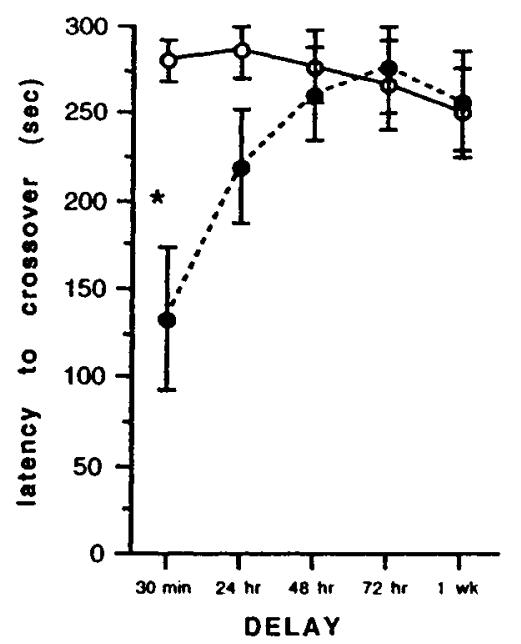

B.

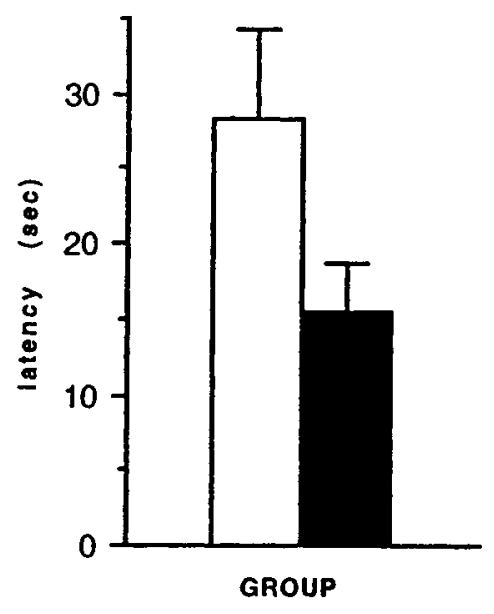

c.

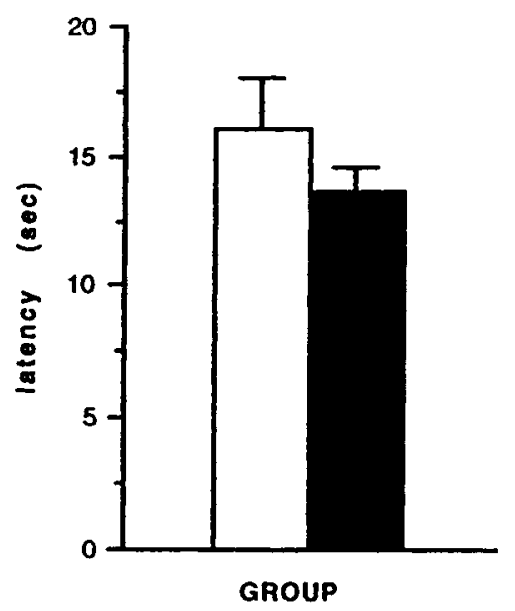

Figure 2. Results of passive-avoidance retention trials and behavioral control tests. In all three panels, the open bars or circles represent vehicle-infused rats $(n=12)$, and the dark bars or circles represent azide-infused rats $(n=11)$. (A) Latency to cross over into the dark chamber as a function of delay after the acquisition trial (*significantly different from vehicle latency, $p<.05$ ); note that data are presented in their quantitative form but were analyzed nonparametrically on the basis of ranks. (B) Latency to first crossover as a control for spontaneous tendency to cross over. (C) Hot-plate test as a control for pain thresholds. 
A.

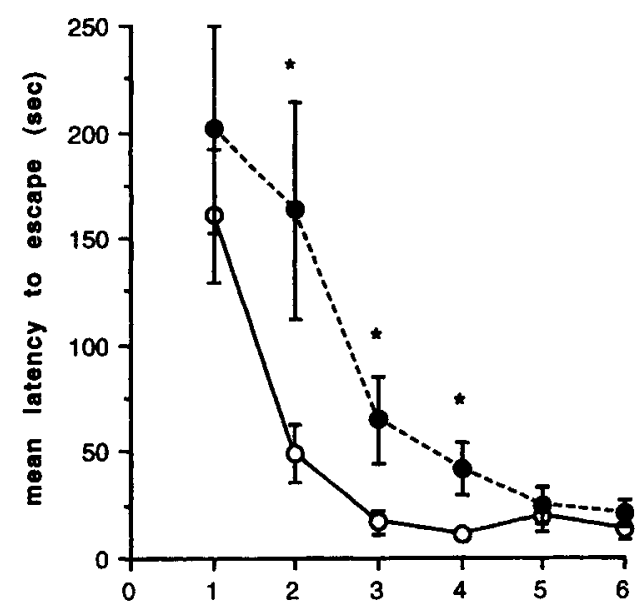

C.

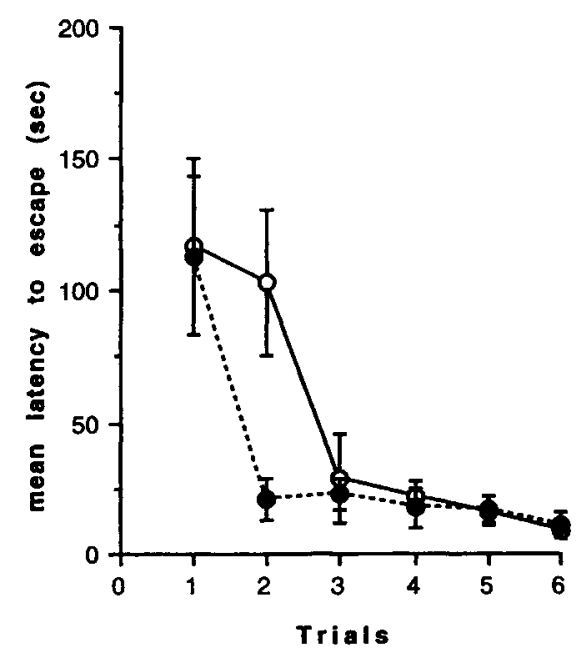

B.

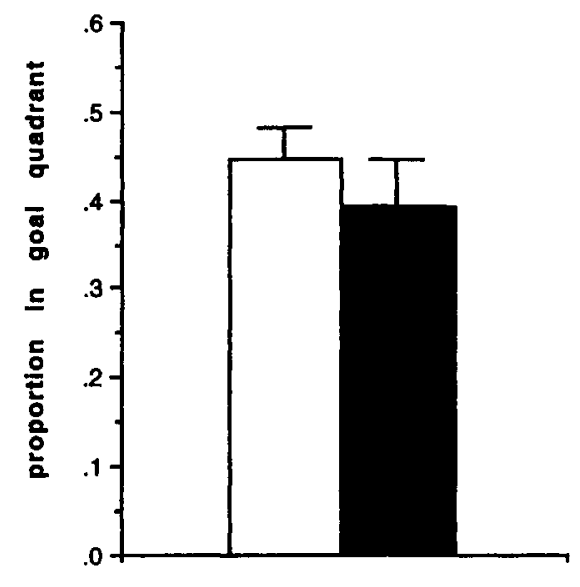

D.

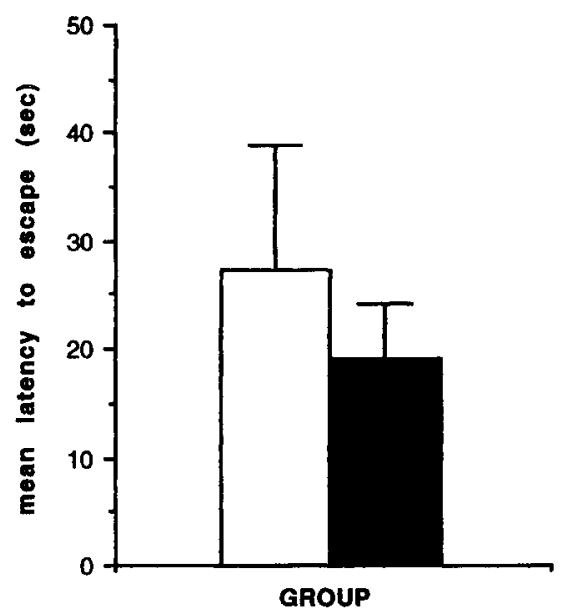

Figure 3. Water-maze performance during infusions. In all panels, the open bars or circles represent vehicle-infused rats, and the dark bars or circles represent azide-infused rats. (A) Water-maze acquisition measured by latency to escape to the hidden goal platform ( $n \mathrm{~s}=8$ vehicle, 7 azide) (*significantly different from vehicle latency, $p<.05$ ). (B) Water-maze retention represented by the proportion of time spent in the goal quadrant during a 30 -sec probe trial $(n s=8$ vehicle, 7 azide). (C) Acquisition of a cued platform $(n s=6$ per group). (D) Swim time to a visible platform ( $n \mathrm{~s}=6$ per group).

azide were able to successfully grasp a rod in their forepaws (raw data and statistics reported in Table 1).

\section{Histological Analysis}

Sections stained with thionin to visualize cell bodies revealed no marked azide-related neuronal loss in any region of the brain at the end of a 28-day infusion of sodium azide. All regions of the brain were examined visually by a blind observer, with special attention given to the hippocampus and neocortex. We cannot rule out the possibility that cell counts would reveal a small but potentially consistent or significant cell loss in one or more brain regions.
Sections stained with silver to visualize normal fibers revealed a subtle decrease in the staining of myelinated nerve fibers in the retrosplenial (Area 29 or posterior cingulate) cortex of some azide-infused animals relative to vehicle-infused animals (data not shown, refer to Experiment 2), which was not significantly different when quantified by densitometry $[t(12)=0.448, p<.662$; see Experiment 2, Figure 8 below for a graphical depiction).

\section{EXPERIMENT 2}

We were interested in determining whether the impairments described in Experiment 1 were transient, relying 


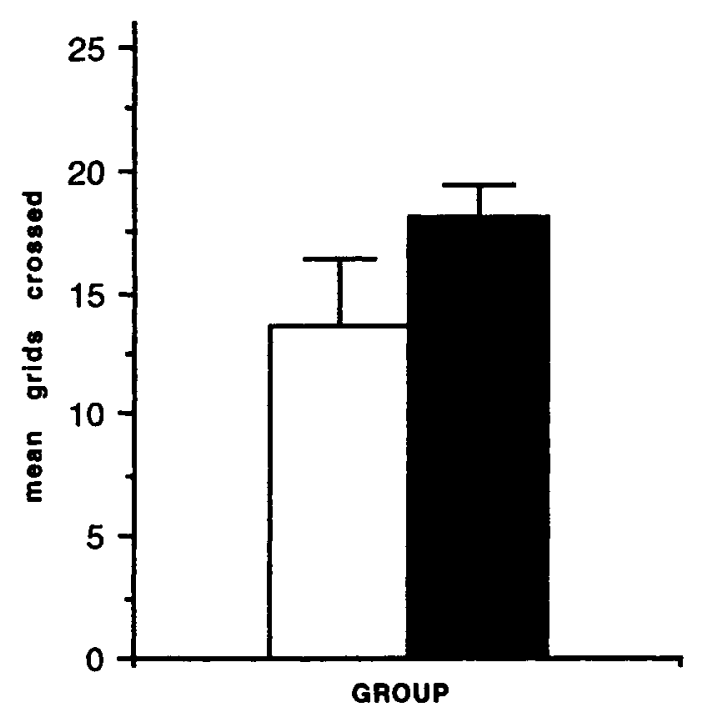

Figure 4. Grid locomotor activity measured during infusions. Activity was measured by the mean number of grids crossed during a 4-min session. The open bars represent vehicle-infused animals $(n=8)$, and the dark bars represent azide-infused animals $(n=7)$.

on the continued presence of toxin, or whether there was damage that extended beyond the duration of the infusion. In Experiment 2, we selected one of the learning tasks used in Experiment 1, the Morris water maze, and tested animals starting either 1 week after (short-term) or 6 months after (long-term) infusions. Because we observed a significant azide-related impairment in fine sensorimotor integration in Experiment 1, we also tested the animals on the locomotor and sensorimotor tests after the termination of infusions.

\section{Method}

\section{Subjects}

The subjects were as described for Experiment 1 .

\section{Surgeries}

Pump implantations for infusion of sodium azide or saline were as described for Experiment 1. On Days 28-30 after the start of infusions, osmotic minipumps were removed under ketamine/xylazine anesthesia (dose and administration as previously described). An incision was made in the right flank just rostral to the minipump, and the pump was removed with a pair of forceps. The wound was closed, and wound clips were removed approximately 1 week later.

\section{Behavioral Testing}

Behavioral testing was initiated either 1 week (short-term postazide) or 6 months (long-term postazide) after minipump removal. All behavioral testing was performed between 1000 and $1600 \mathrm{~h}$ in behavioral testing rooms located within the animal facility, as described for Experiment 1. Figure 5 diagrams testing time lines for Experiments 2 (reversal testing not done) and 3 (locomotor and sensorimotor testing not done).

Water-maze acquisition. Water-maze acquisition was tested using the modification of Morris's water-maze task described for Experiment 1 . All animals were behaviorally naive when tested shortterm postazide. To reduce animal usage, the long-term postazide water-maze animals had received passive-avoidance testing during azide infusion. To control for the possibility that prior experience with passive avoidance affects later water-maze performance differentially for vehicle- versus azide-infused animals, a smaller cohort of behaviorally naive animals ( $n \mathrm{~s}=4$ vehicle, 6 azide) was later tested 6 months postinfusion. At each time point, a 6 (trial) $\times$ 2 (infusion) mixed factorial ANOVA was performed to compare learning in the azide-infused groups with that in the vehicle-infused controls, and a Tukey HSD post hoc test was used to further evaluate differences between groups.

Water-maze retention. Water-maze retention was tested and analyzed as described for Experiment 1.

Locomotion. The animals were tested for locomotor activity on a grid as described for Experiment 1. The short-term postazide animals were tested 8 weeks after pump removal, and the long-term postazide animals were tested 8 months after pump removal. Data were analyzed with a Student's independent-groups $t$ test at each time point.

Sensorimotor integration tests. The animals were evaluated for fine sensorimotor integration and motor coordination as described for Experiment 1. These tests were conducted either 8 weeks or 8 months after pump removal. A Fisher's exact test for contingency tables was conducted for each measure at each time point.

Histology. Brain tissue was collected for histological processing either 8 weeks or 8 months after pump removal. Tissue was collected, processed, and analyzed as described for Experiment 1.

\section{Results}

\section{Behavior}

Water maze. Short-term postazide, there was a trend toward a water-maze acquisition deficit $[F(1,120)=2.715, p>$ .09 ; data not shown]; however, by 6 months posttreatment, no acquisition deficit was apparent in azide-treated animals relative to controls $[F(1,90)=0.185, p>.66$; data not shown]. The behaviorally naive 6 -month postazide cohort,

Table 1

Frequency Table of Rats' Sensorimotor Integration Abilities by Group and Time After Infusion

\begin{tabular}{|c|c|c|c|}
\hline \multirow[b]{2}{*}{ Group } & \multirow{2}{*}{$\frac{\text { Experiment } 1}{\text { During Infusion }}$} & \multicolumn{2}{|c|}{ Experiment 2} \\
\hline & & Short-Term After & Long-Term After \\
\hline \multicolumn{4}{|c|}{ Gross Sensorimotor Coordination (Edge Reaching) } \\
\hline $\begin{array}{l}\text { Vehicle-infused } \\
\text { Azide-infused }\end{array}$ & $\begin{array}{l}6 \text { of } 8 \text { successful } \\
6 \text { of } 7 \text { successful }\end{array}$ & $\begin{array}{l}8 \text { of } 8 \text { successful } \\
8 \text { of } 8 \text { successful }\end{array}$ & $\begin{array}{l}7 \text { of } 7 \text { successful } \\
9 \text { of } 9 \text { successful }\end{array}$ \\
\hline \multicolumn{4}{|c|}{ Fine Sensorimotor Coordination (Rod Grasping) } \\
\hline $\begin{array}{l}\text { Vehicle-infused } \\
\text { Azide-infused }\end{array}$ & $\begin{array}{l}8 \text { of } 8 \text { successful } \\
3 \text { of } 7 \text { successful* }\end{array}$ & $\begin{array}{l}8 \text { of } 8 \text { successful } \\
8 \text { of } 8 \text { successful }\end{array}$ & $\begin{array}{l}6 \text { of } 7 \text { successful } \\
9 \text { of } 9 \text { successful }\end{array}$ \\
\hline
\end{tabular}

*Azide-infused rats performed significantly worse than did vehicle-infused rats, according to Fisher's exact test $(p<.03)$. 
A

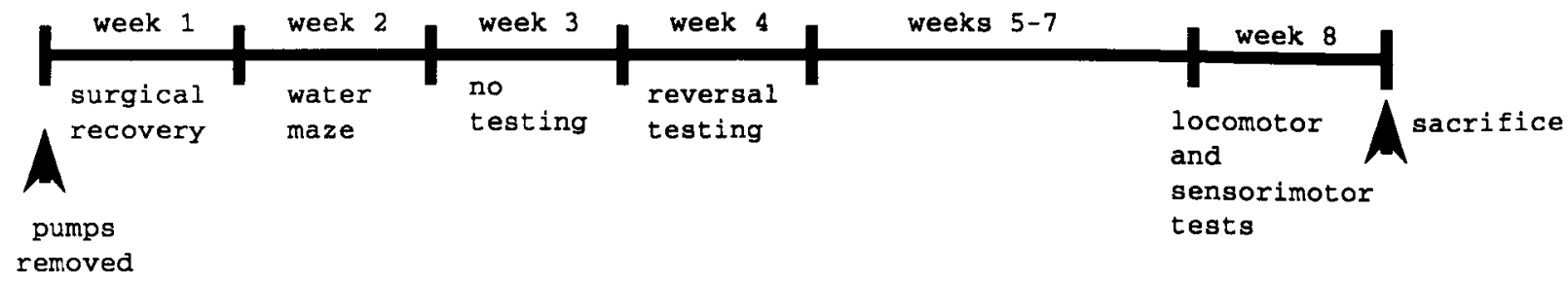

B

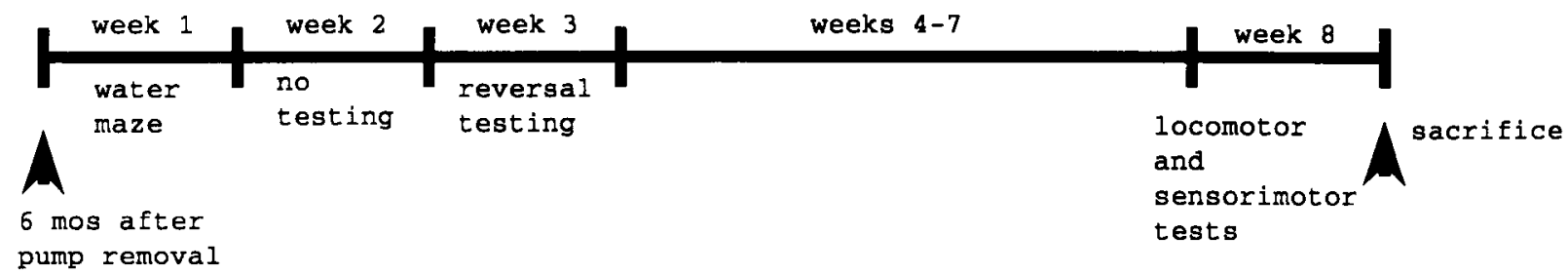

Figure 5. Time lines for behavioral evaluation of animals after azide infusions. The arrow on each time line indicates time "zero." (A) Behavioral testing short-term postinfusion. (B) Behavioral testing long-term postinfusion.

run to control for possible effects of prior exposure to passive avoidance, also showed no acquisition deficits relative to vehicle controls $[F(1,40)=0.004, p>.95$; data not shown]. As observed during azide infusion, there was no deficit in memory for the escape platform location after full acquisition at either time point postinfusion [short-term postinfusion, $t(20)=0.065, p>.94$; long-term postinfusion, $t(15)=0.303, p>.76$; additional long-term cohort, $t(8)=0.936, p>.37$; data not shown].

Locomotion and sensorimotor integration. Gridcrossing measures revealed no significant difference in locomotor activity between vehicle- and azide-infused rats at either postazide time point [short-term postinfusion $t(14)=1.044, p>.31$; long-term postinfusion, $t(14)=0.119, p>.90$; data not shown]. In addition, rats from both groups at both time points were able to demonstrate normal gross sensorimotor coordination by reaching for an edge with their paws and normal fine sensorimotor coordination by grasping a rod in their forepaws (data reported in Table 1). Therefore, the fine sensorimotor deficit detected during azide infusions appeared to resolve shortly after the termination of infusions.

\section{Histological Analysis}

Similar to observations made during azide infusions, an examination of sections stained with thionin to visualize cell bodies revealed no apparent azide-related neuronal loss in any region of the brain at either postazide time point. As stated for Experiment 1, we cannot rule out the possibility that cell counts would reveal a small but potentially significant cell loss.

Sections stained with silver to visualize normal fibers again revealed some azide-related changes. Shortly after termination of infusions ( 8 weeks), a marked alteration in fiber staining was observed in the retrosplenial cortex of azide-infused animals relative to vehicle-infused controls (Figures 6A and 6B). Specifically, there was a pronounced loss of myelinated fiber staining in the granular retrosplenial cortex and in the presubicular cortex near its juncture with the retrosplenial cortex. Within both of these areas, there was a dramatic decrease in myelinated fiber staining in Layers III and IV-most notably, in aggregated bundles of radial fibers that run perpendicular to the cortical surface. In contrast, there was an apparently complete preservation of tangentially oriented myelinated fibers in Layer I. In addition, argyrophilic debris was often noted in the area of myelinated fiber loss.

Some apparent decrease in staining of myelin or disorganization of the myeloarchitecture was also noted in some areas of frontal cortex and the subiculum (data not shown). However, the observed changes were subtle and inconsistent relative to those seen in the retrosplenial cortex. Most areas of neocortex showed no appreciable change in myeloarchitecture, and no changes in nerve fiber density or organization were evident in the hippocampus proper. By 8 months postinfusion, changes in the myelin architecture of the retrosplenial cortex were no longer evident (Figures 6C and 6D), suggesting that structural changes in the retrosplenial cortex were reversed during the period of behavioral recovery from the azide-induced impairments. Quantification of the fiber densities confirmed that there was a significant loss of fiber staining in the retrosplenial cortex of azide-infused animals shortterm postinfusion $[t(10)=3.318, p<.008$; Figure 7], which recovered by long-term postinfusion $[t(10)=$ $0.237, p>.818$; Figure 7]. 

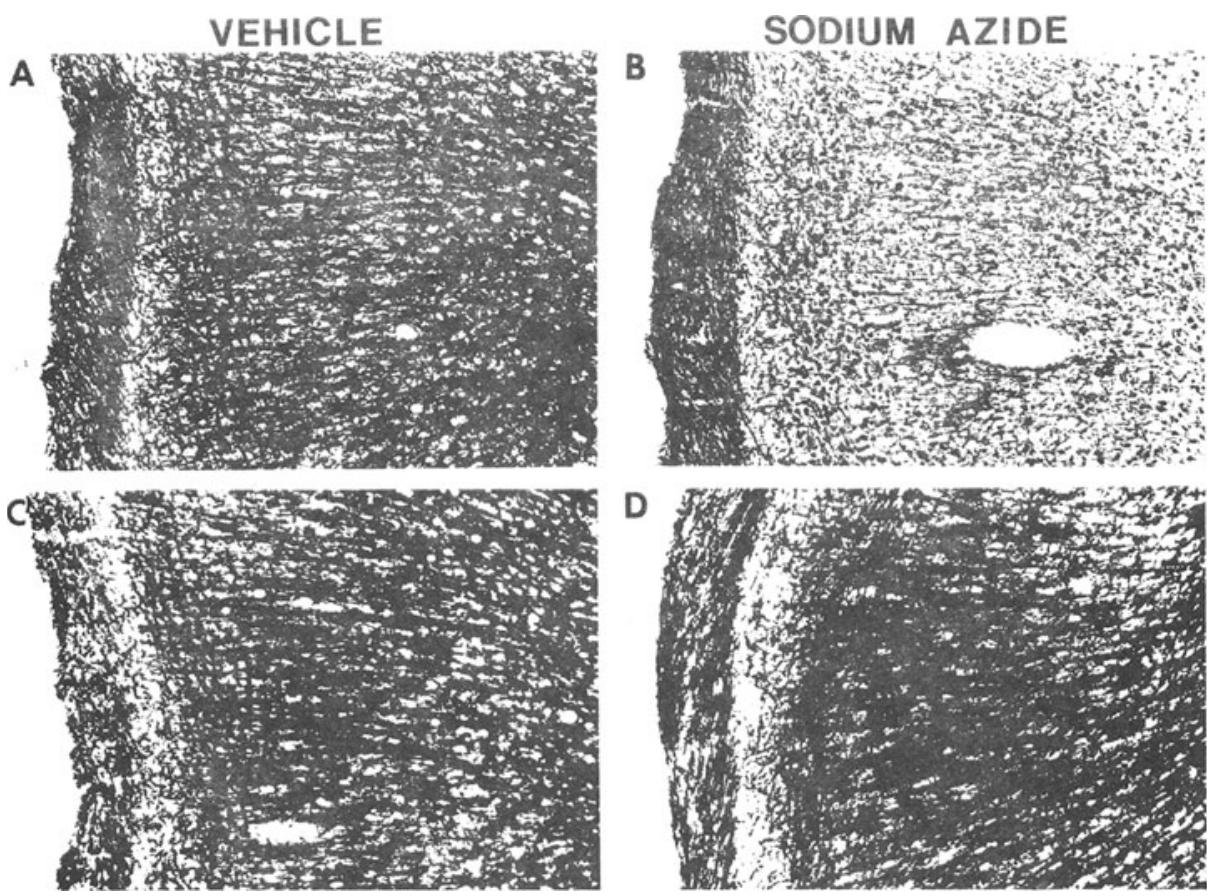

Figure 6. Photomicrographs of silver-stained sections of Area 29 of the retrosplenial cortex. Sections from azide-infused rats are to be compared only with those of vehicle-infused rats at the same time point due to variability in silver impregnation from run to run. Scale bar $=0.25 \mathrm{~mm}$. (A) Vehicle-infused rat sacrificed short-term postinfusion. (B) Azide-infused rat sacrificed short-term postazide. (C) Vehicleinfused rat sacrificed long-term postazide. (D) Azide-infused rat sacrificed long-term postazide.

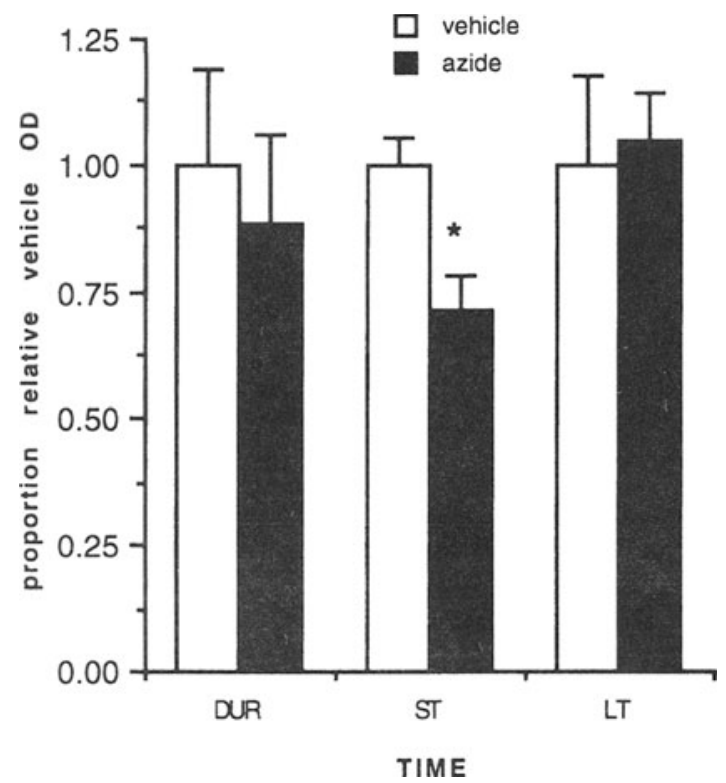

Figure 7. Retrosplenial cortex myelinated fiber densities. Densities are normalized to occipital cortex values for each animal and are shown as the proportion of vehicle values to facilitate comparisons across time points. DUR $=$ during infusions $(n=6$ vehicle, $n=8$ azide); $S T=$ short-term postinfusions $(n=6$ vehicle, $n=6$ azide); LT $=$ long-term postinfusions $(n=6$ vehicle, $n=6$ azide) ( ${ }^{\star}$ significantly different from vehicles, $p<.05$ ).

\section{EXPERIMENT 3}

The loss of myelinated fiber staining in the retrosplenial cortex combined with the finding of a statistical trend toward a water-maze acquisition deficit in animals shortterm postazide suggested that some degree of learning impairment might still be present after the termination of azide infusions. To test this hypothesis, the complexity of the water-maze task was increased by exposing postazide animals to a water-maze reversal task 1 week after the original water-maze task.

\section{Method \\ Subjects \\ The subjects were as described for Experiment 1 .}

\section{Surgeries}

Pump implantations and removals were as described for Experiments 1 and 2.

\section{Behavioral Testing}

Reversal testing was initiated 1 week after the initial water-maze spatial probe trial. All behavioral testing was performed between 1000 and $1600 \mathrm{~h}$ in behavioral testing rooms located within the animal facility, as described for Experiment 1. Figure 5 diagrams behavioral testing protocols for Experiments 2 (reversal testing not done) and 3 (locomotor and sensorimotor testing not done).

After postinfusion animals had acquired the goal platform location in the water-maze task, they received a 1 -week delay and were 
A.

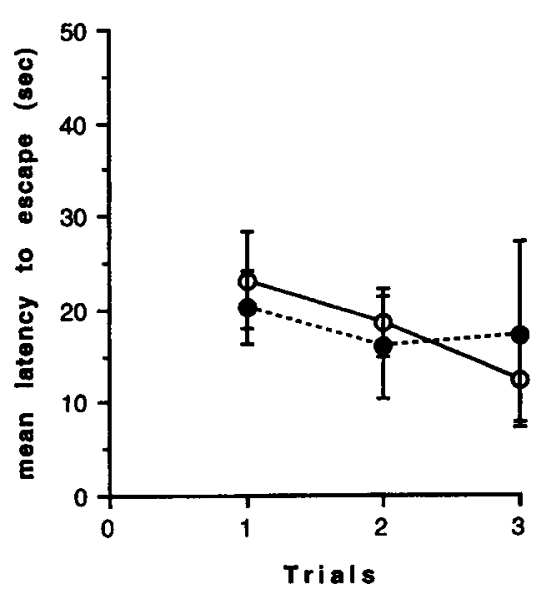

C.



B.

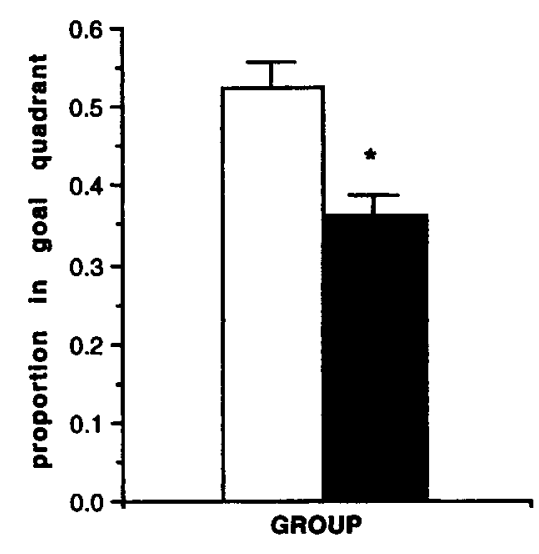

D.

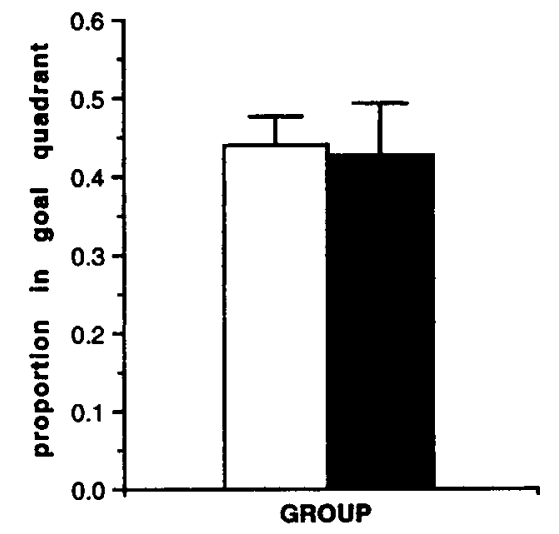

Figure 8. Water-maze reversals. In all panels, the open bars or circles represent vehicle-infused rats $(n=8$, both time points), and the dark bars or circles represent azide-infused rats $(n=8$ shortterm, $n=9$ long-term). (A) Reversal acquisition short-term postinfusions measured by latency to escape to the hidden goal platform. (B) Reversal retention short-term postinfusions represented by the proportion of time spent in the goal quadrant during a 30-sec probe trial ("significantly different from vehicle score, $p<.05$ ). (C) Reversal acquisition long-term postinfusions. (D) Reversal retention long-term postinfusions.

then reintroduced to the water maze with the hidden escape platform reversed to the diagonally opposite quadrant. The animals received three daily reversal acquisition trials with the platform position reversed and were then given a probe trial to assess retention on the 4th day. Water-maze reversals were carried out both shortterm and long-term postazide, to see whether the added complexity of this manipulation would reveal a deficit at either of these time points. The analysis of reversal acquisition was performed with a 3 (trial) $\times 2$ (infusion) mixed factorial ANOVA. The analysis of reversal retention was performed for the probe trial using Student's independent-groups $t$ tests to compare vehicle- and azide-infused groups.

\section{Results}

When the animals learned the initial platform location 1 week after pump removal and were reversed beginning 1 week after their spatial probe trial, they showed no im- pairment in performance of the reversal task $[F(1,84)=$ $.004, p>.95$; Figure $8 \mathrm{~A}]$ but showed a significant impairment during the spatial probe trial for retention $[t(14)=3.901, p<.002$; Figure $8 \mathrm{~B}]$. This reversal retention impairment was no longer present 6 months after infusion $[t(15)=0.198, p>.84$; Figure $8 \mathrm{D}]$.

\section{DISCUSSION}

\section{General Conclusions}

The present results confirm previous findings of learning and memory impairments during sodium-azide infusion (Bennett et al., 1992; Bennett \& Rose, 1992) and extend those findings to include retention of a fully acquired water-maze task and passive-avoidance retention. In addition, we find memory impairments that outlast 
azide infusion but that recover by 6 months postinfusion. Finally, we have discovered a loss of myelinated fiber staining in the retrosplenial cortex of azide-infused animals, which is most striking shortly after the termination of infusions.

\section{Passive-Avoidance Deficit}

Relative to vehicle-infused rats, azide-infused rats showed a significant retention deficit after a one-trial passive-avoidance acquisition. This finding expands Bennett et al.'s (1992) finding of active-avoidance deficits to include passive avoidance. Our data suggest, however, that the azide-induced passive-avoidance deficit may represent delayed consolidation rather than no memory processing, because azide-infused animals actually show improved retention with increased delays. This improvement is not likely to be accounted for by repeated exposures to the task, because the animals were not shocked when they crossed to the dark side of the chamber during any of the retention trials. They would therefore have no obvious reason to learn to avoid the dark chamber on the basis of any experience except for the initial one-trial acquisition.

\section{Water Mazes}

The present water-maze data are consistent with the idea that azide-infused rats show delayed, but eventually good, retention of learned information. Azide-infused rats eventually acquired the water-maze task, but their learning curves revealed delayed learning relative to vehicleinfused rats. Because we used a water-maze protocol that allowed azide-infused rats to learn the task relatively quickly, we were able to demonstrate that, once azideinfused animals had acquired a water-maze task, they had no difficulty exhibiting memory for the platform location. This result does not contradict Bennett and Rose's (1992) finding of a retention deficit, because their azideinfused rats had not acquired the water-maze task when the spatial probe trial was performed. Although it is possible that the delayed acquisition resulted from a performance deficit, this explanation seems unlikely. Azideinfused animals acquired a cued-platform water-maze task at least as well as vehicle-infused animals did and also swam as quickly to a visible escape platform. Hippocampal lesions also produce impairments on the spatial version of the water maze, without causing impairments in cue learning (Morris, Garrud, Rawlins, \& O'Keefe, 1982).

Postinfusion testing demonstrated that the water-maze acquisition deficit detected during azide infusion improves quickly after infusions are terminated and recovers completely by 6 months after infusions. Because there was still a trend toward a deficit shortly after the termination of infusions, we reversed the platform location to see whether or not the addition of a more difficult manipulation would reveal a statistically significant impairment. Although the acquisition of the reversal did not appear to be impaired in the azide-infused animals, their retention scores on the spatial probe trial were significantly worse than the scores of the vehicle-infused animals. No reversal deficit was detected 6 months after infusions, when azide-related retrosplenial cortex pathology had also disappeared.

\section{Relationship to Alzheimer's Disease}

Overall, the behavioral data suggest that azide-infused animals have difficulty exhibiting rapid and efficient learning of new tasks and that, after azide infusions, they still exhibit some deficits that eventually recover. Delayed acquisition, like that described in our rats during azide infusion, is not characteristic of the memory difficulties observed in late-stage AD (Kopelman, 1985) or after massive cell loss in the hippocampus (Jarrard, 1980; Morris et al., 1982). After hippocampal lesions or during latestage $\mathrm{AD}$, full acquisition of spatial or declarative (in humans) information is rarely achieved. In addition, retention deficits are apparent in these conditions, especially with increasing delays. Instead, the deficits we observe are more reminiscent of problems found when the hippocampus and limbic cortex are not communicating with one another properly, such as in the early stages of $A D$ and with certain lesions or pharmacological disruptions of areas connected to the hippocampus or of hippocampal efferent pathways (Jarrard, 1980; Kopelman, 1985; Skelton \& McNamara, 1992; Storandt, Botwinick, \& Danzifer, 1984). That is, the animals are capable of learning new tasks but show delayed learning and difficulty with more complex tasks.

Among the earliest histological deficits observed in $\mathrm{AD}$ are tangles in Layer II of the entorhinal cortex, the primary cortical projection area to the hippocampus (Braak \& Braak, 1991; Hof et al., 1992). This pathology appears to be associated with impaired information transfer from the entorhinal cortex to the hippocampus through the perforant path and a loss of perforant path synapses into the hippocampus. In more intermediate stages of $A D$, cells of the hippocampus and some of its afferents, such as the basal forebrain cholinergic system, are lost (Braak \& Braak, 1991; Davis \& Maloney, 1976; deLeon et al., 1993; Whitehouse et al., 1982). During the later stages of $\mathrm{AD}$, the loss of cells within the hippocampus results in reduced outflow from the hippocampus into direct or indirect hippocampal efferents, such as the subicular and retrosplenial cortices. During the latest stages of $\mathrm{AD}$, tangles, amyloid plaques, and biochemical abnormalities can be found in all of these areas of the cortex (Braak \& Braak, 1991; Chun, Gui-zhen, \& Braak, 1994; Vogt, Crino, \& Vogt, 1992; Vogt, Van Hoesen, \& Vogt, 1990).

Our histological finding of altered myeloarchitecture in the retrosplenial cortex, but not the entorhinal cortex or hippocampus, suggests that azide infusion does not produce a deficit that closely models typical cases of AD. That is, in most cases of $A D$, damage to the retrosplenial cortex occurs late in the disease and is thus compromised only after damage has occurred in the entorhinal cortex and hippocampus (Braak \& Braak, 1991). It is the combined damage to these, as well as other, areas of the brain 
that results in the profound memory impairment of latestage AD. The damage we observed after azide infusions is, in some respects, the obverse of that normally observed during AD. We could find no apparent abnormalities in the entorhinal cortex or the hippocampus but rather found damage in areas of the cortex that are damaged later in $\mathrm{AD}$ and are among those areas that eventually receive hippocampal input. Specifically, we found that the fiber tracts in the subicular and parasubicular cortex, the hippocampus' first projection into the cortex, appeared to be mildly disorganized. More notably, we found that the fibers in the retrosplenial cortex, to which the subiculum, presubiculum, parasubiculum, and postsubiculum project (Finch, Derian, \& Babb, 1984; Meibach \& Siegel, 1977; Swanson \& Cowan, 1977; van Groen \& Wyss, 1990; Vogt \& Miller, 1983 ), were profoundly affected.

Although the retrosplenial cortex becomes involved relatively late in the disease progression in most $A D$ patients, recent reports suggest that the retrosplenial or posterior cingulate cortex is more involved earlier in the disease in certain subtypes of AD. Specifically, AD patients that present with Balint's syndrome (visual disturbances), rather than with dementia, show earlier retrosplenial and parietal involvement and less frontal involvement than do most patients (Hof et al., 1993). Perhaps of more interest is the recent finding that very old $A D$ patients (in their $90 \mathrm{~s}$ and $100 \mathrm{~s}$ ) show a unique pattern of plaque and tangle deposition that favors cortical areas more often showing late involvement (Giannakopoulos et al., 1995). In these patients, the retrosplenial cortex shows much higher than usual plaque and tangle deposition, and, for plaques, only the retrosplenial/posterior cingulate and superior parietal cortical pathology predicts memory score. Finally, although the retrosplenial/posterior cingulate cortex does not acquire plaques and tangles until late in the disease process of most $\mathrm{AD}$ patients, positron emission tomography scans have shown a severe decrease in energy metabolism very early in most patients with probable AD (Minoshima, Foster, \& Kuhl, 1994). Therefore, it is possible that the damage to the retrosplenial cortex after azide infusions may be relevant to some aspects of $\mathrm{AD}$.

\section{The Retrosplenial Cortex and Other Pathology}

In addition to $\mathrm{AD}$, other human pathologies are characterized by involvement of the retrosplenial cortex. For instance, patients with Pick's disease show a deposition of Pick's bodies in many of the same areas of the cortex, including the retrosplenial cortex, as do AD patients (Yoshimura, 1989). Human diseases in which there is a loss of myelin or myelinated fibers, such as amyotrophic lateral sclerosis, also show retrosplenial abnormalities (Kew et al., 1993). The pathology in these two diseases, however, is neither selective nor preferential for the retrosplenial cortex. The most common causes of selective or preferential retrosplenial damage or dysfunction are localized infarcts in the retrosplenial cortex or its connections. In these cases, memory impairments are often se- vere. For instance, T.R., a patient with relatively selective damage to the retrosplenial cortex after a subarachnoid hemorrhage, showed profound memory disturbances, including both retrograde and anterograde amnesia (Valenstein et al., 1987).

In addition, a selective dysregulation of metabolism in the retrosplenial cortex has been reported with mercury toxicity (O'Carroll, Masterton, Dougall, Ebmeier, \& Goodwin, 1995), in which loss of cortical myelin has also been detected by MRI (White, Feldman, Moss, \& Proctor, 1993). Recent studies of workers with low levels of chronic occupational mercury exposure (mercury miners, dentists, factory workers using mercury components) have shown that the most significant, and dose-/exposurerelated, consequence of their exposure is memory impairment (Kishi et al., 1994; Ngim, Foo, Boey, \& Jeyaratnam, 1992; Ritchie et al., 1995; Smith, Langolf, \& Goldberg, 1983; Soleo, Urbano, Petrera, \& Ambrosi, 1990; Triebig \& Schaller, 1982; Williamson, Teo, \& Sanderson, 1982). Impairment is most often reported for short-term memory, cognitive flexibility, and cognitive tracking. Our findings of more severe impairments at shorter delays (passive avoidance) and with increased demand for cognitive flexibility (water-maze reversals) suggest the possibility that mercury and azide toxicity could have some similar anatomical bases. Interestingly, one group used the Sternberg memory test to measure speed of memory processing after chronic low-level mercury exposure and found that the speed of memory processing was significantly impaired (Williamson et al., 1982). This finding is consistent with our proposal that animals receiving azide infusions show delayed memory processing. It would be interesting to more directly determine whether or not mercury exposure produced a loss of retrosplenial myelinated fibers. Unfortunately, studies of mercury toxicity in animals have used prenatal exposure and, despite the promising findings of passiveavoidance deficits and abnormalities of cortical neurochemistry, may be difficult to directly relate to adult toxicity (Cagiano et al., 1990; Zanoli, Truzzi, Veneri, Braghiroli, \& Baraldi, 1994).

One study of a pathological state in adult rats has shown an indirect relationship between retrosplenial pathology and memory impairments. The normal age-related breakdown of retrosplenial dendritic fibers is accelerated in spontaneously hypertensive rats (SHR), such that they show this pathology by 12 months of age (Wyss \& van Groen, 1991). Early impairments in SHR rat radialarm-maze performance parallel this fiber pathology (Wyss, Fisk, \& van Groen, 1992). Chronic treatment with antihypertensives from 3-12 months of age results in an alleviation of the learning impairment normally seen in SHR rats at 12 months. Although hypertension has not been directly shown to produce retrosplenial cortex damage and memory impairments, the results of this study suggest such a possibility. Hypertension in humans has been correlated with increased memory impairment and increased occurence of white matter lesions (Pathy, 1988; 
Schmidt et al., 1991; Wilkie \& Eisdorfer, 1971). Any relationships between these conditions and retrosplenial disorganization have yet to be determined.

\section{The Retrosplenial Cortex and Learning}

In addition to its involvement in various pathological conditions, the retrosplenial cortex has also been shown to be involved in normal learning and memory in both human and animal studies. Human studies have shown changes in retrosplenial cortex metabolism by PET during various learning and memory tasks. Specifically, the retrosplenial cortex shows decreased activity during nonspatial verbal memory (Swartz et al., 1994), the right retrosplenial cortex shows increased metabolism during the presentation of novel versus familiar stimuli (Tulving et al., 1994), and the left retrosplenial cortex shows increased metabolism during eyeblink conditioning (Molchan et al., 1994).

Nictitating-membrane conditioning in rabbits is very similar to eyeblink conditioning in humans. In rabbits, lesions of the retrosplenial cortex produce no deficit in learning the initial task but produce an impairment in reversal of the association to another tone (Berger et al., 1986). This observation is consistent with our finding short-term postazide of little difficulty in learning of the platform location, but significant impairment in reversing the platform location. Further evidence for a role of the retrosplenial cortex in reversal learning is the finding that ibotenic acid lesions of the retrosplenial cortex in mice result in poor acquisition and initial reversals of a T-maze spatial discrimination task (Meunier, Jaffard, \& Destrade, 1991). In addition, these mice showed deficits in long-term retention of T-maze reversals.

An intensive and elegant set of studies of the role of the retrosplenial cortex in active-avoidance learning in rabbits has been completed by Gabriel and colleagues. These electrophysiological studies have helped to elucidate the entire learning system, including the retrosplenial cortex, involved in acquiring this task. For example, serial lesions have shown that the subiculum is necessary for training-related activity (TIA) in the retrosplenial cortex, which is necessary for TIA in the anterior cingulate (Gabriel \& Sparenborg, 1987). In addition, they have found that retrosplenial cortex lesions suppress anterior thalamic TIA and suppress conditioned responses in rabbits (Gabriel, Sparenborg, \& Stolar, 1987), suggesting that the retrosplenial cortex may activate areas necessary for the behavioral responses in avoidance performance. The retrosplenial cortex itself shows discriminative TIA (which differentiates between tones predictive of shock; Gabriel, Vogt, Kubota, Poremba, \& Kang, 1991), as well as selective responses to familiarity versus novelty (Gabriel, 1990).

The retrosplenial cortex has also been shown to be involved in spatial learning and memory in rats. Bilateral aspiration lesions of the retrosplenial cortex result in acquisition and retention deficits in the water maze (Kolb \& Whishaw, 1991; Sutherland, Whishaw, \& Kolb, 1988). An experiment that combined a unilateral hippocampal lesion with a contralateral retrosplenial cortex lesion demonstrated that the hippocampus and retrosplenial cortex work together during water-maze acquisition (Sutherland \& Hoesing, 1993). The combined lesion severely disrupted water-maze acquisition, whereas each lesion alone had little or no effect. The water-maze acquisition impairment that we observed during azide is less severe than those observed with either bilateral hippocampal or retrosplenial lesions. In contrast with these lesion studies, we do not observe appreciable neuronal loss in these areas, so our relatively mild impairments are not surprising. Rather than causing severe or extensive damage, azide infusions may be creating a partial and transient disconnection of the retrosplenial cortex from other components of the limbic system and neocortex.

Neurochemical manipulations specific to the retrosplenial cortex have also been shown to cause behavioral impairments. Specifically, depletions of cholecystokinin result in impaired visual discrimination performance in mice (Meziane, Devigne, Tramu, \& Soumireu-Mourat, 1993), muscarinic receptor antagonism causes delayed water-maze acquisition in rats (Riekkinen, Kuitunen, \& Riekkinen, 1995), and norepinephrine depletion decreases early avoidance responses in rabbits (Sparenborg \& Gabriel, 1992). Although we see no significant loss of myelinated fibers in the retrosplenial cortex during azide infusions or 8 months postinfusion, we have not analyzed the retrosplenial cortex neurochemically at these time points. It is possible that reductions in some neurotransmitters or peptides could be present in the retrosplenial cortex, hippocampus, or other parts of the limbic circuitry before, during, or after our most striking retrosplenial myelinated fiber loss.

In the present study, we have demonstrated learning and memory impairments and an accompanying disruption of retrosplenial cortex myeloarchitecture after azide infusions. It is probable, however, that azide infusions result in additional abnormalities that remain to be described. For example, the original work of Bennett et al. (1992) demonstrates that azide treatment produces an impairment in the maintenance of long-term potentiation in the hippocampus, where we found no obvious cell or fiber loss. Such findings suggest that physiological, neurochemical, or subtle structural properties of other portions of the limbic circuitry are also compromised in this model. In fact, the impairments we observe during azide infusions may be a result of these more subtle abnormalities. Further research will be necessary to fully characterize all of the disruptions in memory circuitry that might result from sodium-azide infusions.

\section{REFERENCES}

Beal, M. F., Hyman, B. T., \& Koroshetz, W. (1993). Do defects in mitochondrial energy metabolism underlie the pathology of neurodegenerative diseases? Trends in Neurosciences, 16, 125-131.

Bennett, M. C., Diamond, D. M., Stryker, S. L., Parks, J. K., \& PARKER, W. D. (1992). Cytochrome oxidase inhibition: A novel animal model of Alzheimer's disease. Journal of Geriatric Psychiatry \& Neurology, 5, 93-101. 
Bennett, M. C., \& Rose, G. M. (1992). Chronic sodium azide treatment impairs learning of the Morris water-maze task. Behavioral \& Neural Biology, 58, 72-75.

Berger, T. W., Weikart, C. L., Bassett, J. L., \& OrR, W. B. (1986) Lesions of the retrosplenial cortex produce deficits in reversal learning of the rabbit nictitating membrane response: Implications for potential interactions between hippocampal and cerebellar brain systems. Behavioral Neuroscience, 100, 802-809.

Blackstad, T. W., Heimer, L., \& Mugnaini, E. (1981). Experimental neuroanatomy: General approaches and laboratory procedures. In L. Heimer \& M. J. Robards (Eds.), Neuroanatomical tract-tracing methods (pp. 1-54). New York: Plenum.

BRAAK, H., \& BRAAK, E. (1991). Neuropathological staging of Alzheimerrelated changes. Acta Neuropathologica, 82, 239-259.

Cagiano, R., DeSalvia, M. A., Renna, G., Tortella, E., Braghiroli, D., Parenti, C., Zanoli, P., Baraldi, M., Annau, Z., \& Cuomo, V. (1990). Evidence that exposure to methyl mercury during gestation induces behavioral and neurochemical changes in offspring of rats. Neurotoxicology \& Teratology, 12, 23-28.

Carlesimo, G. A., \& Oscar-Berman, M. (1992). Memory deficits in Alzheimer's patients: A comprehensive review. Neuropsychology Reviews, 3, 119-169.

Chun, M., Gui-zhen, W., \& Braak, H. (1994). Pathological changes of the retrosplenial cortex in senile dementia of Alzheimer type. Chinese Medical Journal, 107, 119-123.

Davis, P., \& Maloney, A. J. (1976). Selective loss of cholinergic neurons in Alzheimer's disease. Lancet, 2, 1403.

deleon, M. J., Golomb, J., George, A. E., Convit, A., Tarshish, C. Y., McRae, T., DeSanti, S., Smith, G., Ferris, S. H., Noz, M., \& RusinEK, H. (1993). The radiologic prediction of Alzheimer disease: The atrophic hippocampal formation. American Journal of Neuroradiology, 14, 897-906.

Finch, D. M., Derian, E. L., \& BabB, T. L. (1984). Afferent fibers to rat cingulate cortex. Experimental Neurology, 83, 468-485.

GabriEL, M. (1990). Functions of anterior and posterior cingulate cortex during avoidance learning in rabbits. Progress in Brain Research, $85,467-483$

Gabriel, M., \& Sparenborg, S. (1987). Posterior cingulate cortical lesions eliminate learning-related unit activity in the anterior cingulate cortex. Brain Research, 409, 151-157.

Gabriel, M., Sparenborg, S. P., \& Stolar, N. (1987). Hippocampal control of cingulate cortical and anterior thalamic information processing during learning in rabbits. Experimental Brain Research, 67, $131-152$.

Gabriel, M., Vogt, B. A., Kubota, Y., Poremba, A., \& Kang, E. (1991). Training-stage related neuronal plasticity in limbic thalamus and cingulate cortex during learning: A possible key to mnemonic retrieval. Behavioural Brain Research, 46, 175-185.

Giannakopoulos, P., Hof, P. R., Giannakopoulos, A.-S., HerrMann, F. R., Michel, J.-P., \& Bouras, C. (1995). Regional distribution of neurofibrillary tangles and senile plaques in the cerebral cortex of very old patients. Archives of Neurology, 52, 1150-1159.

Hof, P. R., Archin, N., Osmand, A. P., Dougherty, J. H., Wells, C., Bouras, C., \& Morrison, J. H. (1993). Posterior cortical atrophy in Alzheimer's disease: Analysis of a new case and re-evaluation of a historical report. Acta Neuropathologica, 86, 215-223.

Hof, P. R., Bierer, L. M., Perl, D. P., Delacourt, A., Buee, L., Bouras, C., \& Morrison, J. H. (1992). Evidence of early vulnerability of the medial and inferior aspects of the temporal lobe in an 82 year old patient with preclinical signs of dementia. Archives of $\mathrm{Neu}$ rology, 49, 946-953.

JARRARD, L. E. (1980). Selective hippocampal lesions and behavior. Physiological Psychology, 8, 198-206.

Jorm, A. F., Korten, A. E., \& Henderson, A. S. (1987). The prevalence of dementia: A quantitative integration of the literature. Acta Psychiatra Scandinavica, 76, 465-479.

Kew, J. J. M., Leigh, P. N., Playford, E. D., Passingham, R. E., GoldStein, L. H., Frackowiak, R. S. J., \& Brooks, D. J. (1993). Cortical function in amyotrophic lateral sclerosis. Brain, 116, 655-680.

Kish, S. J., Bergeron, C., Rajput, A., Dozic, S., Mastrogiacomo, F., Chang, L.-J., Wilson, J. M., DiStefano, L. M., \& Nobrega, J. N.
(1992). Brain cytochrome oxidase in Alzheimer's disease. Journal of Neurochemistry, 59, 776-779.

Kishi, R., Doi, R., Fukuchi, Y., Satoh, H., Satoh, T., OnO, A., MorIwaka, F., Tashiro, K., Takahata, N., Sasatani, H., ShiraKaShi, H., Kamada, T., \& NaKagawa, K. (1994). Residual neurobehavioural effects associated with chronic exposure to mercury vapor. Occupational \& Environmental Medicine, 51, 35-41.

KolB, B., \& Whishaw, I. Q. (1991). Mechanisms underlying behavioral sparing after neonatal retrosplenial cingulate lesions in rats: Spatial navigation, cortical architecture, and electroencephalographic activity. Brain Dysfunction, 4, 75-92.

Kopelman, M. D. (1985). Multiple memory deficits in Alzheimer-type dementia: Implications for pharmacotherapy. Psychological Medicine, 15, 527-541.

MeibaCh, R. C., \& SiEgel, A. (1977). Subicular projections to the posterior cingulate cortex in rats. Experimental Neurology, 57, 264-274.

Meunier, M., Jaffard, R., \& Destrade, C. (1991). Differential involvement of anterior and posterior cingulate cortices in spatial discriminative learning in a T-maze in mice. Behavioural Brain Research, 44, 133-143.

Meziane, H., Devigne, C., Tramu, G., \& Soumireu-Mourat, B. (1993). Effects of anti-CCK-8 antiserum on acquisition and retrieval by mice in an appetitive task. Peptides, 14, 67-73.

Minoshima, S., Foster, N. L., \& Kuhl, D. E. (1994). Posterior cingulate cortex in Alzheimer's disease. Lancet, 344, 895.

Molchan, S. E., Sunderland, T., Mcintosh, A. R., Herscovitch, P. \& SCHREURS, B. G. (1994). A functional anatomical study of associative learning in humans. Proceedings of the National Academy of Sciences, 91, 8122-8126.

Morris, R. G. M. (1981). Spatial localization does not require the presence of local cues. Learning \& Motivation, 12, 239-260.

Morris, R. G. M., Garrud, P., Rawlins, J. N. P., \& O'Keefe, J. (1982). Place navigation impaired in rats with hippocampal lesions. Nature, 297, 681-683.

Ngim, C. H., Foo, S. C., Boey, K. W., \& Jeyaratnam, J. (1992). Chronic neurobehavioural effects of elemental mercury in dentists. British Journal of Industrial Medicine, 49, 782-790.

o'Carroll, R. E., Masterton, G., Dougall, N., Ebmeier, K. P., \& Goodwin, G. M. (1995). The neuropsychiatric sequelae of mercury poisoning: The Mad Hatter's disease revisited. British Journal of Psychiatry, 167, 95-98.

Parker, W. D., Filley, C. M., \& Parks, J. K. (1990). Cytochrome oxidase deficiency in Alzheimer's disease. Neurology, 40, 1302-1303.

PATHY, S. J. (1988). Hypertension and associated diseases in elderly patients. Journal of Hypertension, 6, S37-S40.

Riekkinen, P., Kuitunen, J., \& Riekkinen, M. (1995). Effects of scopolamine infusions into the anterior and posterior cingulate on passive avoidance and water maze navigation. Brain Research, 685, 46-54.

Ritchie, K. A., Macdonald, E. B., Hammersley, R., O’Neil, J. M., McGowan, D. A., Dale, I. M., \& Wesnes, K. (1995). A pilot study of the effect of low level exposure to mercury on the health of dental surgeons. Occupational \& Environmental Medicine, 52, 813-817.

Schmidt, R., Fazekas, F., OfFenbacher, H., Lytwyn, H., Blematl, B., Niederkorn, K., Horner, S., Payer, F., \& Freidl, W. (1991). Magnetic resonance imaging white matter lesions and cognitive impairment in hypertensive individuals. Archives of Neurology, 48, $417-420$.

Simonian, N. A., \& Hyman, B. T. (1993). Functional alterations in Alzheimer's disease: Diminution of cytochrome oxidase in the hippocampal formation. Journal of Neuropathology \& Experimental Neurology, 52, 580-585.

Skelton, R. W., \& McNamara, R. K. (1992). Bilateral knife cuts to the perforant path disrupt spatial learning in the Morris water maze. Hippocampus, 2, 73-80.

Smith, P. J., LANGolf, G. D., \& Goldberg, J. (1983). Effects of occupational exposure to elemental mercury on short term memory. British Journal of Indusirial Medicine, 40, 413-419.

Soleo, L., Urbano, M. L., Petrera, V., \& Ambrosi, L. (1990). Effects of low exposure to inorganic mercury on psychological performance. British Journal of Industrial Medicine, 47, 105-109.

Sparenborg, S., \& Gabriel, M. (1992). Local norepinephrine deple- 
tion and learning-related neuronal activity in cingulate cortex and anterior thalamus of rabbits. Experimental Brain Research, 92, 267-285.

Storandt, M., Botwinick, J., \& DanZifer, W. L. (1984). Psychometric differentiation of mild senile dementia of the Alzheimer type. Archives of Neurology, 41, 497-499.

Sutherland, R. J., \& Hoesing, J. M. (1993). Posterior cingulate cortex and spatial memory: A microlimnology analysis. In B. A. Vogt \& M. Gabriel (Eds.), Neurobiology of cingulate cortex and limbic thalamus (pp. 461-477). Boston: Birkhauser.

Sutherland, R. J., Whishaw, I. Q., \& Kolb, B. (1988). Contributions of cingulate cortex to two forms of spatial learning and memory. Journal of Neuroscience, 8, 1863-1872.

SWANSON, L. W., \& CoWAN, W. M. (1977). An autoradiographic study of the efferent connections of the hippocampal formation in the rat. Journal of Comparative Neurology, 172, 49-84.

Swartz, B. E., Halgren, E., Fuster, J., \& Mandel Kern, M. (1994). An ${ }^{18}$ FDG-PET study of cortical activation during a short-term visual memory task in humans. NeuroReport, 5, 925-928.

TRIEBIG, G., \& SCHALler, K.-H. (1982). Neurotoxic effects in mercuryexposed workers. Behavioural Toxicology \& Teratology, 4, 717-720.

Tulving, E., Markowitsch, H. J., Kapur, S., Habib, R., \& Houle, S. (1994). Novelty encoding networks in the human brain: Positron emission tomography data. NeuroReport, 5, 2525-2528.

Valenstein, E., Bowers, D., Verfaellie, M., Heilman, K. M., DAY, A., \& WATSON, R. T. (1987). Retrosplenial amnesia. Brain, 110, $1631-1646$.

van Groen, T., \& Wyss, J. M. (1990). The postsubicular cortex in the rat: Characterization of the fourth region of the subicular cortex and its connections. Brain Research, 529, 165-177.

Vogt, B. A., Crino, P. B., \& Vogt, L. J. (1992). Reorganization of cingulate cortex in Alzheimer's disease: Neuron loss, neuritic plaques, and muscarinic receptor binding. Cerebral Cortex, 2, 526-535.

VOGT, B. A., \& MilLER, M. W. (1983). Cortical connections between rat cingulate cortex and visual, motor, and postsubicular cortices. Journal of Comparative Neurology, 216, 192-210.
Vogt, B. A., Van Hoesen, G. W., \& Vogt, L. J. (1990). Laminar distribution of neuron degeneration in posterior cingulate cortex in Alzheimer's disease. Acta Neuropathologica, 80, 581-589.

Watson, R. E., Wiegand, S. J., Clogh, R. W., \& Hoffman, G. E. (1986). Use of cryprotectant to maintain long-term peptide immunoreactivity and tissue morphology. Peptides, 7, 155-159.

White, R. F., Feldman, R. G., Moss, M. B., \& Proctor, S. P. (1993). Magnetic resonance imaging (MRI), neurobehavioral testing, and toxic encephalopathy: Two cases. Environmental Research, 61, 117-123.

Whitehouse, P. J., Price, D. L., Struble, R. G., Clark, A. W., COYLE, J. T., \& DeLonG, M. R. (1982). Alzheimer's disease and senile dementia: Loss of neurons in the basal forebrain. Science, 215 , 1237-1239.

WILKIE, F., \& EISDORFER, C. (1971). Intelligence and blood pressure in the aged. Science, 172, 959-962.

Williamson, A. M., Teo, R. K. C., \& Sanderson, J. (1982). Occupational mercury exposure and its consequences for behavior. International Archives of Occupational \& Environmental Health, 50, 273-286.

WySs, J. M., FISK, G., \& VAN GROEN, T. (1992). Impaired learning and memory in mature spontaneously hypertensive rats. Brain Research, 592, 135-140.

Wyss, J. M., \& VAN Groen, T. (1991). Age related breakdown of dendritic bundles in the retrosplenial cortex of the rat. Society for Neuroscience Abstracts, 17, 128.

Yoshimura, N. (1989). Topography of Pick body distribution in Pick's disease: A contribution to understanding the relationship between Pick's and Alzheimer's diseases. Clinical Neuropathology, 8, 1-6.

Zanoli, P., Truzzi, C., Veneri, C., Braghiroli, D., \& Baraldi, M. (1994). Methyl mercury during late gestation affects temporarily the development of cortical muscarinic receptors in rat offspring. Pharmacology \& Toxicology, 75, 261-264.

(Manuscript received April 4, 1996; revision accepted for publication September 1, 1996.) 